\title{
GENERALIZED OSTROWSKI TYPE INEQUALITIES FOR FUNCTIONS WHOSE LOCAL FRACTIONAL DERIVATIVES ARE GENERALIZED $s$-CONVEX IN THE SECOND SENSE
}

\author{
Hüseyin Budak ${ }^{1}$, Mehmet Zeki Sarikaya ${ }^{2}$,Erhan Set ${ }^{3}$ \\ 1,2 Department of Mathematics, Faculty of Science and Arts, Düzce University, Düzce-Turkey \\ ${ }^{3}$ Department of Mathematics, Faculty of Arts and Sciences, Ordu University, 52200, Ordu, Turkey \\ hsyn.budak@gmail.com,sarikayamz@gmail.com,erhanset@yahoo.com \\ Received: 14 March 2016; accepted: 15 September 2016
}

\begin{abstract}
In this paper, we establish some generalized Ostrowski type inequalities for functions whose local fractional derivatives are generalized $s$-convex in the second sense.

Keywords: generalized Hermite-Hadamard inequality, generalized Hölder inequality, generalized convex functions
\end{abstract}

\section{Introduction}

In 1938, Ostrowski established the following interesting integral inequality for differentiable mappings with bounded derivatives [1]:

Theorem 1. (Ostrowski inequality) Let $f:[a, b] \rightarrow R$ be a differentiable mapping on $(a, b)$ whose derivative $f^{\prime}:(a, b) \rightarrow R$ is bounded on $(a, b)$, i.e. $\left\|f^{\prime}\right\|_{\infty}:=\sup _{t \in(a, b)}\left|f^{\prime}(t)\right|<\infty$. Then, we have the inequality

$$
\left|f(x)-\frac{1}{b-a} \int_{a}^{b} f(t) d t\right| \leq\left[\frac{1}{4}+\frac{\left(x-\frac{a+b}{2}\right)^{2}}{(b-a)^{2}}\right](b-a)\left\|f^{\prime}\right\|_{\infty},
$$

for all $x \in[a, b]$. The constant $\frac{1}{4}$ is the best possible.

In recent years, the fractal theory has received significant attention. The calculus on the fractal set can lead to better comprehension for the various real world models from science and engineering [2-19].

The purpose of this paper is to establish some local fractional integral inequalities using generalized $s$-convex in the second sense on real linear fractal set $\mathrm{R}^{\alpha}(0<\alpha<1)$. This paper is divided into the following three sections. In Section 2, we give the definitions of the local fractional derivatives and local fractional 
integrals and introduce several useful notations on fractal space which will be used our main results. In Section 3, the main results are presented.

\section{Preliminaries}

Recall the set $R^{\alpha}$ of real line numbers and use the Gao-Yang-Kang's idea to describe the definition of the local fractional derivative and local fractional integral, see $[14,15]$ and so on.

Recently, the theory of Yang's fractional sets [yang] was introduced as follows. For $0<\alpha \leq 1$, we have the following $\alpha$-type set of element sets:

$Z^{\alpha}:$ The $\alpha$-type set of integer is defined as the set $\left\{0^{\alpha}, \pm 1^{\alpha}, \pm 2^{\alpha}, \ldots, \pm n^{\alpha}, \ldots\right\}$.

$Q^{\alpha}:$ The $\alpha$-type set of the rational numbers is defined as the set $\left\{m^{\alpha}=\left(\frac{p}{q}\right)^{\alpha}\right.$ : $p, q \in Z, q \neq 0\}$.

$J^{\alpha}:$ The $\alpha$-type set of the irrational numbers is defined as the set $\left\{m^{\alpha} \neq\left(\frac{p}{q}\right)^{\alpha}: p, q \in Z, q \neq 0\right\}$.

$R^{\alpha}:$ The $\alpha$-type set of the real line numbers is defined as the set $R^{\alpha}=Q^{\alpha} \cup J^{\alpha}$.

If $a^{\alpha}, b^{\alpha}$ and $c^{\alpha}$ belongs the set $R^{\alpha}$ of real line numbers, then

(1) $a^{\alpha}+b^{\alpha}$ and $a^{\alpha} b^{\alpha}$ belongs the set $R^{\alpha}$;

(2) $a^{\alpha}+b^{\alpha}=b^{\alpha}+a^{\alpha}=(a+b)^{\alpha}=(b+a)^{\alpha}$;

(3) $a^{\alpha}+\left(b^{\alpha}+c^{\alpha}\right)=(a+b)^{\alpha}+c^{\alpha}$;

(4) $a^{\alpha} b^{\alpha}=b^{\alpha} a^{\alpha}=(a b)^{\alpha}=(b a)^{\alpha}$;

(5) $a^{\alpha}\left(b^{\alpha} c^{\alpha}\right)=\left(a^{\alpha} b^{\alpha}\right) c^{\alpha}$;

(6) $a^{\alpha}\left(b^{\alpha}+c^{\alpha}\right)=a^{\alpha} b^{\alpha}+a^{\alpha} c^{\alpha}$;

(7) $a^{\alpha}+0^{\alpha}=0^{\alpha}+a^{\alpha}=a^{\alpha}$ and $a^{\alpha} 1^{\alpha}=1^{\alpha} a^{\alpha}=a^{\alpha}$.

The definition of the local fractional derivative and local fractional integral can be given as follows.

Definition 1. [14] A non-differentiable function $f: R \rightarrow R^{\alpha}, x \rightarrow f(x)$ is called to be local fractional continuous at $x_{0}$, if for any $\varepsilon>0$, there exists $\delta>0$, such that

$$
\left|f(x)-f\left(x_{0}\right)\right|<\varepsilon^{\alpha}
$$

holds for $\left|x-x_{0}\right|<\delta$, where $\varepsilon, \delta \in R$. If $f(x)$ is local continuous on the interval $(a, b)$, we denote $f(x) \in C_{\alpha}(a, b)$. 
Definition 2. [14] The local fractional derivative of $f(x)$ of order $\alpha$ at $x=x_{0}$ is defined by

$$
f^{(\alpha)}\left(x_{0}\right)=\left.\frac{d^{\alpha} f(x)}{d x^{\alpha}}\right|_{x=x_{0}}=\lim _{x \rightarrow x_{0}} \frac{\Delta^{\alpha}\left(f(x)-f\left(x_{0}\right)\right)}{\left(x-x_{0}\right)^{\alpha}}
$$

where $\Delta^{\alpha}\left(f(x)-f\left(x_{0}\right)\right) \cong \Gamma(\alpha+1)\left(f(x)-f\left(x_{0}\right)\right)$.

If there exists $f^{(k+1) \alpha}(x)=\overbrace{D_{x}^{\alpha} \ldots D_{x}^{\alpha}}^{k+1 \text { times }} f(x)$ for any $x \in I \subseteq R$, then we denoted $f \in D_{(k+1) \alpha}(I)$, where $k=0,1,2, \ldots$.

Definition 3. [14] Let $f(x) \in C_{\alpha}[a, b]$. Then the local fractional integral is defined by,

$$
{ }_{a} I_{b}^{\alpha} f(x)=\frac{1}{\Gamma(\alpha+1)} \int_{a}^{b} f(t)(d t)^{\alpha}=\frac{1}{\Gamma(\alpha+1)} \lim _{\Delta t \rightarrow 0} \sum_{j=0}^{N-1} f\left(t_{j}\right)\left(\Delta t_{j}\right)^{\alpha},
$$

with $\Delta t_{j}=t_{j+1}-t_{j}$ and $\Delta t=\max \left\{\Delta t_{1}, \Delta t_{2}, \ldots, \Delta t_{N-1}\right\}$, where $\left[t_{j}, t_{j+1}\right], j=0, \ldots, N-1$ and $a=t_{0}<t_{1}<\ldots<t_{N-1}<t_{N}=b$ is a partition of interval $[a, b]$.

Here, it follows that ${ }_{a} I_{b}^{\alpha} f(x)=0$ if $a=b$ and ${ }_{a} I_{b}^{\alpha} f(x)={ }_{b} I_{a}^{\alpha} f(x)$ if $a<b$. If for any $x \in[a, b]$, there exists ${ }_{a} I_{x}^{\alpha} f(x)$, then we denote by $f(x) \in I_{x}^{\alpha}[a, b]$.

Lemma 1. [14]

(1) (Local fractional integration is anti-differentiation) Suppose that $f(x)=g^{(\alpha)}(x) \in C_{\alpha}[a, b]$, then we have

$$
{ }_{a} I_{b}^{\alpha} f(x)=g(b)-g(a) .
$$

(2) (Local fractional integration by parts) Suppose that $f(x), g(x) \in D_{\alpha}[a, b]$ and $f^{(\alpha)}(x), g^{(\alpha)}(x) \in C_{\alpha}[a, b]$, then we have

$$
{ }_{a} I_{b}^{\alpha} f(x) g^{(\alpha)}(x)=\left.f(x) g(x)\right|_{a} ^{b}-{ }_{a} I_{b}^{\alpha} f^{(\alpha)}(x) g(x) .
$$

Lemma 2. [14]

$$
\begin{gathered}
\frac{d^{\alpha} x^{k \alpha}}{d x^{\alpha}}=\frac{\Gamma(1+k \alpha)}{\Gamma(1+(k-1) \alpha)} x^{(k-1) \alpha} ; \\
\frac{1}{\Gamma(\alpha+1)} \int_{a}^{b} x^{k \alpha}(d x)^{\alpha}=\frac{\Gamma(1+k \alpha)}{\Gamma(1+(k+1) \alpha)}\left(b^{(k+1) \alpha}-a^{(k+1) \alpha}\right), k \in R .
\end{gathered}
$$


Lemma 3. (Generalized Hölder's inequality) [14] Let $f, g \in C_{\alpha}[a, b], \quad p, q>1$ with $\frac{1}{p}+\frac{1}{q}=1$, then

$\frac{1}{\Gamma(\alpha+1)} \int_{a}^{b}|f(x) g(x)|(d x)^{\alpha} \leq\left(\frac{1}{\Gamma(\alpha+1)} \int_{a}^{b}|f(x)|^{p}(d x)^{\alpha}\right)^{\frac{1}{p}}\left(\frac{1}{\Gamma(\alpha+1)} \int_{a}^{b}|g(x)|^{q}(d x)^{\alpha}\right)^{\frac{1}{q}}$.

In [7], the authors introduced two kinds of generalized $s$-convex functions on fractal sets $\mathrm{R}^{\alpha}(0<\alpha<1)$ as follows:

Definition 4. Let $\mathrm{R}_{+}=[0,+\infty)$. A function $f: \mathrm{R}_{+} \rightarrow \mathrm{R}^{\alpha}$ is said to be generalized $s$-convex $(0<s<1)$ in the first sense, if

$$
f\left(\lambda_{1} u+\lambda_{2} v\right) \leq \lambda_{1}^{s \alpha} f(u)+\lambda_{2}^{s \alpha} f(v)
$$

for all $u, v \in \mathrm{R}_{+}$and $\lambda_{1}, \lambda_{2} \geq 0$ with $\lambda_{1}^{s}+\lambda_{2}^{s}=1$. We denote this by $f \in G K_{s}^{1}$.

Definition 5. A function $f: \mathrm{R}_{+} \rightarrow \mathrm{R}^{\alpha}$ is said to be generalized $s$-convex $(0<s<1)$ in the second sense, if

$$
f\left(\lambda_{1} u+\lambda_{2} v\right) \leq \lambda_{1}^{s \alpha} f(u)+\lambda_{2}^{s \alpha} f(v)
$$

for all $u, v \in \mathrm{R}_{+}$and $\lambda_{1}, \lambda_{2} \geq 0$ with $\lambda_{1}+\lambda_{2}=1$. We denote this by $f \in G K_{s}^{2}$.

If we have the reverse inequality, then $f$ is called s-concave.

Sarikaya and Budak proved the following generalized Ostrowski inequality in [10]:

Theorem 2. (Generalized Ostrowski inequality) Let $I \subseteq \mathrm{R}$ be an interval, $f: I^{0} \subseteq \mathrm{R} \rightarrow \mathrm{R}^{\alpha} \quad\left(I^{0}\right.$ is the interior of $\left.I\right)$ such that $f \in D_{\alpha}\left(I^{0}\right)$ and $f^{(\alpha)} \in C_{\alpha}[a, b]$ for $a, b \in I^{0}$ with $a<b$. Then, for all $x \in[a, b]$, we have the identity

$$
\left|f(x)-\frac{\Gamma(1+\alpha)}{(b-a)^{\alpha}}{ }_{a} I_{b}^{\alpha} f(t)\right| \leq 2^{\alpha} \frac{\Gamma(1+\alpha)}{\Gamma(1+2 \alpha)}\left[\frac{1}{4^{\alpha}}+\left(\frac{x-\frac{a+b}{2}}{b-a}\right)^{2 \alpha}\right](b-a)^{\alpha}\left\|f^{(\alpha)}\right\|_{\infty} .
$$

In [8], Mo and Sui established the following Hermite-Hadamard inequality for generalized $s$-convex functions on a real linear fractal set $\mathrm{R}^{\alpha}(0<\alpha<1)$ :

Theorem 3. Suppose that $f: \mathrm{R}_{+} \rightarrow \mathrm{R}^{\alpha}$ is a generalized $s$-convex function in the second sense, where $s \in(0,1)$. Let $a, b \in[0, \infty), a<b$. If $f \in C_{\alpha}[a, b]$, then the following inequalities hold: 


$$
\frac{2^{(s-1) \alpha}}{\Gamma(1+\alpha)} f\left(\frac{a+b}{2}\right) \leq \frac{{ }_{a} I_{b}^{\alpha} f(t)}{(b-a)^{\alpha}} \leq \frac{\Gamma(1+s \alpha)}{\Gamma(1+(s+1) \alpha)}(f(a)+f(b)) .
$$

If $f$ is a generalized $s$-concave, then we have the reverse inequality.

\section{Main results}

We will start with a generalized identity for local fractional integrals:

Theorem 4. Let $I \subseteq R$ be an interval, $f: I^{0} \subseteq \mathrm{R}_{+} \rightarrow \mathrm{R}^{\alpha}$ ( $I^{0}$ is the interior of $I$ ) such that $f \in D_{\alpha}\left(I^{0}\right)$ and $f^{(\alpha)} \in C_{\alpha}[a, b]$ for $a, b \in I^{0}$ with $a<b$. Then, we have the identity

$$
\begin{aligned}
& f(x)-\frac{\Gamma(1+\alpha)}{(b-a)^{\alpha}}{ }_{a} I_{b}^{\alpha} f(t) \\
= & \frac{(x-a)^{2 \alpha}}{(b-a)^{\alpha} \Gamma(1+\alpha)} \int_{0}^{1} t^{\alpha} f^{(\alpha)}(t x+(1-t) a)(d t)^{\alpha} \\
& -\frac{(b-x)^{2 \alpha}}{(b-a)^{\alpha} \Gamma(1+\alpha)} \int_{0}^{1} t^{\alpha} f^{(\alpha)}(t x+(1-t) b)(d t)^{\alpha}
\end{aligned}
$$

for all $x \in[a, b]$.

Proof. Using the local fractional integration by parts (Lemma 1), we have

$$
\begin{aligned}
K_{1}= & \frac{1}{\Gamma(1+\alpha)} \int_{0}^{1} t^{\alpha} f^{(\alpha)}(t x+(1-t) a)(d t)^{\alpha} \\
= & \left.\frac{t^{\alpha} f(t x+(1-t) a)}{(x-a)^{\alpha}}\right|_{0} ^{1} \\
& -\frac{\Gamma(1+\alpha)}{(x-a)^{\alpha}} \Gamma(1+\alpha) \int_{0}^{1} f(t x+(1-t) a)(d t)^{\alpha} \\
= & \frac{f(x)}{(x-a)^{\alpha}}-\frac{\Gamma(1+\alpha)}{(x-a)^{\alpha} \Gamma(1+\alpha)} \int_{0}^{1} f(t x+(1-t) a)(d t)^{\alpha} \\
= & \frac{f(x)}{(x-a)^{\alpha}}-\frac{\Gamma(1+\alpha)}{(x-a)^{2 \alpha} \Gamma(1+\alpha)} \int_{a}^{x} f(u)(d u)^{\alpha} .
\end{aligned}
$$


Similarly, we have

$$
\begin{aligned}
& K_{2}=\frac{1}{\Gamma(1+\alpha)} \int_{0}^{1} t^{\alpha} f^{(\alpha)}(t x+(1-t) b)(d t)^{\alpha} \\
= & \frac{f(x)}{(b-x)^{\alpha}}+\frac{\Gamma(1+\alpha)}{(b-x)^{2 \alpha} \Gamma(1+\alpha)} \int_{x}^{b} f(u)(d u)^{\alpha} .
\end{aligned}
$$

Using (4) and (5), we obtain

$$
\begin{aligned}
& \frac{(x-a)^{2 \alpha}}{\Gamma(1+\alpha)} \int_{0}^{1} t^{\alpha} f^{(\alpha)}(t x+(1-t) a)(d t)^{\alpha} \\
& -\frac{(b-x)^{2 \alpha}}{\Gamma(1+\alpha)} \int_{0}^{1} t^{\alpha} f^{(\alpha)}(t x+(1-t) b)(d t)^{\alpha} \\
= & (x-a)^{2 \alpha} K_{1}-(b-x)^{2 \alpha} K_{2} \\
= & (b-a)^{\alpha} f(x)-\frac{\Gamma(1+\alpha)}{\Gamma(1+\alpha)} \int_{a}^{b} f(u)(d u)^{\alpha} \\
= & (b-a)^{\alpha} f(x)-\Gamma(1+\alpha){ }_{a} I_{b}^{\alpha} f(u)
\end{aligned}
$$

which is the required result.

Theorem 5. The assumptions of Theorem 4 are satisfied. If $\left|f^{(\alpha)}\right|$ is generalized $s$-convex in the second sense on $[a, b]$ for some fixed $s \in(0,1)$, then we have the inequality

$$
\begin{aligned}
& \left|f(x)-\frac{\Gamma(1+\alpha)}{(b-a)^{\alpha}}{ }_{a} I_{b}^{\alpha} f(t)\right| \\
& \leq 2^{\alpha}\left(\frac{\Gamma(1+s \alpha)}{\Gamma(1+(s+1) \alpha)}\right)\left[\frac{1}{4^{\alpha}}+\left(\frac{x-\frac{a+b}{2}}{b-a}\right)^{2 \alpha}\right](b-a)^{\alpha}\left\|f^{(\alpha)}\right\|_{\infty}
\end{aligned}
$$

for all $x \in[a, b]$ where $\left\|f^{(\alpha)}\right\|_{\infty}:=\sup _{t \in(a, b)}\left|f^{(\alpha)}(t)\right|$.

Proof. By Theorem 4 and since $\left|f^{(\alpha)}\right|$ is generalized s-convex in the second sense, then we have 


$$
\begin{aligned}
& \left|f(x)-\frac{\Gamma(1+\alpha)}{(b-a)^{\alpha}}{ }_{a} I_{b}^{\alpha} f(t)\right| \\
& \leq \frac{(x-a)^{2 \alpha}}{(b-a)^{\alpha} \Gamma(1+\alpha)} \int_{0}^{1} t^{\alpha}\left|f^{(\alpha)}(t x+(1-t) a)\right|(d t)^{\alpha} \\
& +\frac{(b-x)^{2 \alpha}}{(b-a)^{\alpha} \Gamma(1+\alpha)} \int_{0}^{1} t^{\alpha}\left|f^{(\alpha)}(t x+(1-t) b)\right|(d t)^{\alpha} \\
& \leq \frac{(x-a)^{2 \alpha}}{(b-a)^{\alpha} \Gamma(1+\alpha)} \int_{0}^{1} t^{\alpha}\left[t^{\alpha s}\left|f^{(\alpha)}(x)\right|+(1-t)^{\alpha s}\left|f^{(\alpha)}(a)\right|\right](d t)^{\alpha} \\
& +\frac{(b-x)^{2 \alpha}}{(b-a)^{\alpha} \Gamma(1+\alpha)} \int_{0}^{1} t^{\alpha}\left[t^{\alpha s}\left|f^{(\alpha)}(x)\right|+(1-t)^{\alpha s}\left|f^{(\alpha)}(b)\right|\right](d t)^{\alpha} \\
& \leq \frac{\left\|f^{(\alpha)}\right\|_{\infty}}{(b-a)^{\alpha}}\left[(x-a)^{2 \alpha}+(b-x)^{2 \alpha}\right] \frac{1}{\Gamma(1+\alpha)} \int_{0}^{1}\left[t^{\alpha(s+1)}+t^{\alpha}(1-t)^{\alpha s}\right](d t)^{\alpha} \\
& =\left\|f^{(\alpha)}\right\|_{\infty}\left(\frac{\Gamma(1+s \alpha)}{\Gamma(1+(s+1) \alpha)}\right)\left[\frac{(x-a)^{2 \alpha}+(b-x)^{2 \alpha}}{(b-a)^{\alpha}}\right] \\
& =2^{\alpha}\left(\frac{\Gamma(1+s \alpha)}{\Gamma(1+(s+1) \alpha)}\right)\left[\frac{1}{4^{\alpha}}+\left(\frac{x-\frac{a+b}{2}}{b-a}\right)^{2 \alpha}\right](b-a)^{\alpha}\left\|f^{(\alpha)}\right\|_{\infty} .
\end{aligned}
$$

Here, we used the fact

$$
\frac{1}{\Gamma(1+\alpha)} \int_{0}^{1} t^{\alpha(s+1)}(d t)^{\alpha}=\frac{\Gamma(1+(s+1) \alpha)}{\Gamma(1+(s+2) \alpha)}
$$

and

$$
\frac{1}{\Gamma(1+\alpha)} \int_{0}^{1} t^{\alpha}(1-t)^{\alpha s}(d t)^{\alpha}=\frac{\Gamma(1+s \alpha)}{\Gamma(1+(s+1) \alpha)}-\frac{\Gamma(1+(s+1) \alpha)}{\Gamma(1+(s+2) \alpha)} .
$$

This completes the proof.

Remark 1. If we take $s=1$ in (6), then (6) reduces to (2).

Corollary 1. Under assumption of Theorem 5 with $x=\frac{a+b}{2}$, we have the following midpoint inequality 


$$
\left|f\left(\frac{a+b}{2}\right)-\frac{\Gamma(1+\alpha)}{(b-a)^{\alpha}}{ }_{a} I_{b}^{\alpha} f(t)\right| \leq \frac{(b-a)^{\alpha}}{2^{\alpha}} \frac{\Gamma(1+s \alpha)}{\Gamma(1+(s+1) \alpha)}\left\|f^{(\alpha)}\right\|_{\infty} .
$$

Theorem 6. The assumptions of Theorem 4 are satisfied. If $\left|f^{(\alpha)}\right|^{q}$ is generalized $s$-convex in the second sense on $[a, b]$ for some fixed $s \in(0,1)$, then we have the inequality

$$
\begin{aligned}
& \left|f(x)-\frac{\Gamma(1+\alpha)}{(b-a)^{\alpha}} a_{b}^{\alpha} f(t)\right| \\
& \leq 2^{\left(\frac{q+1}{q}\right) \alpha}\left(\frac{\Gamma(1+p \alpha)}{\Gamma(1+(p+1) \alpha)}\right)^{\frac{1}{p}}\left(\frac{\Gamma(1+s \alpha)}{\Gamma(1+(s+1) \alpha)}\right)^{\frac{1}{q}} \\
& \times\left[\frac{1}{4^{\alpha}}+\left(\frac{x-\frac{a+b}{2}}{b-a}\right)^{2 \alpha}\right](b-a)^{\alpha}\left\|f^{(\alpha)}\right\|_{\infty}
\end{aligned}
$$

for all $x \in[a, b]$ where $p, q>1$ with $\frac{1}{p}+\frac{1}{q}=1$.

Proof. Taking modulus in (3) and using the generalized Hölder's inequality (Lemma 3), we have

$$
\begin{aligned}
& \left|f(x)-\frac{\Gamma(1+\alpha)}{(b-a)^{\alpha}}{ }_{a} I_{b}^{\alpha} f(t)\right| \\
\leq & \frac{(x-a)^{2 \alpha}}{(b-a)^{\alpha} \Gamma(1+\alpha)} \int_{0}^{1} t^{\alpha}\left|f^{(\alpha)}(t x+(1-t) a)\right|(d t)^{\alpha} \\
& +\frac{(b-x)^{2 \alpha}}{(b-a)^{\alpha} \Gamma(1+\alpha)} \int_{0}^{1} t^{\alpha}\left|f^{(\alpha)}(t x+(1-t) b)\right|(d t)^{\alpha} \\
\leq & \frac{(x-a)^{2 \alpha}}{(b-a)^{\alpha}}\left(\frac{1}{\Gamma(1+\alpha)} \int_{0}^{1} t^{p \alpha}(d t)^{\alpha}\right)^{\frac{1}{p}}\left(\frac{1}{\Gamma(1+\alpha)} \int_{0}^{1}\left|f^{(\alpha)}(t x+(1-t) a)\right|^{q}(d t)^{\alpha}\right)^{\frac{1}{q}} \\
& +\frac{(b-x)^{2 \alpha}}{(b-a)^{\alpha}}\left(\frac{1}{\Gamma(1+\alpha)} \int_{0}^{1} t^{p \alpha}(d t)^{\alpha}\right)^{\frac{1}{p}}\left(\frac{1}{\Gamma(1+\alpha)} \int_{0}^{1}\left|f^{(\alpha)}(t x+(1-t) b)\right|^{q}(d t)^{\alpha}\right)^{\frac{1}{q}} .
\end{aligned}
$$

Since $\left|f^{(\alpha)}\right|^{q}$ is generalized s-convex in the second sense on $[a, b]$, then we have 


$$
\begin{aligned}
& \frac{1}{\Gamma(1+\alpha)} \int_{0}^{1}\left|f^{(\alpha)}(t x+(1-t) a)\right|^{q}(d t)^{\alpha} \\
\leq & \frac{1}{\Gamma(1+\alpha)} \int_{0}^{1}\left[t^{\alpha s}\left|f^{(\alpha)}(x)\right|^{q}+(1-t)^{\alpha s}\left|f^{(\alpha)}(a)\right|^{q}\right](d t)^{\alpha} \\
= & \frac{\Gamma(1+s \alpha)}{\Gamma(1+(s+1) \alpha)}\left[\left|f^{(\alpha)}(x)\right|^{q}+\left|f^{(\alpha)}(a)\right|^{q}\right] \\
\leq & 2^{\alpha}\left\|f^{(\alpha)}\right\|_{\infty}^{q} \frac{\Gamma(1+s \alpha)}{\Gamma(1+(s+1) \alpha)},
\end{aligned}
$$

and similarly

$$
\frac{1}{\Gamma(1+\alpha)} \int_{0}^{1}\left|f^{(\alpha)}(t x+(1-t) b)\right|^{q}(d t)^{\alpha} \leq 2^{\alpha}\left\|f^{(\alpha)}\right\|_{\infty}^{q} \frac{\Gamma(1+s \alpha)}{\Gamma(1+(s+1) \alpha)} .
$$

If we substitute the inequality (8) and (9), then we obtain the desired result.

Corollary 2. Under assumption of Theorem 6 with $x=\frac{a+b}{2}$, we have the following midpoint inequality $\left|f(x)-\frac{\Gamma(1+\alpha)}{(b-a)^{\alpha}} a_{b}^{\alpha} f(t)\right| \leq \frac{(b-a)^{\alpha}}{2^{\frac{\alpha}{p}}}\left(\frac{\Gamma(1+p \alpha)}{\Gamma(1+(p+1) \alpha)}\right)^{\frac{1}{p}}\left(\frac{\Gamma(1+s \alpha)}{\Gamma(1+(s+1) \alpha)}\right)^{\frac{1}{q}}\left\|f^{(\alpha)}\right\|_{\infty}^{q}$.

Theorem 7. The assumptions of Theorem 4 are satisfied. If $\left|f^{(\alpha)}\right|^{q}$ is generalized $s$-concave on $[a, b]$ for some fixed $s \in(0,1)$, then we have the inequality

$$
\begin{aligned}
& \left|f(x)-\frac{\Gamma(1+\alpha)}{(b-a)^{\alpha}}{ }_{a} I_{b}^{\alpha} f(t)\right| \\
& \leq \frac{1}{(b-a)^{\alpha}}\left(\frac{2^{(s-1) \alpha}}{\Gamma(1+\alpha)}\right)^{\frac{1}{q}}\left(\frac{\Gamma(1+p \alpha)}{\Gamma(1+(p+1) \alpha)}\right)^{\frac{1}{p}} \\
& \quad \times\left[(x-a)^{2 \alpha}\left|f^{(\alpha)}\left(\frac{a+x}{2}\right)\right|+(b-x)^{2 \alpha}\left|f^{(\alpha)}\left(\frac{b+x}{2}\right)\right|\right]
\end{aligned}
$$

for all $x \in[a, b]$ where $p, q>1$ with $\frac{1}{p}+\frac{1}{q}=1$. 
Proof. From Theorem 4 and using generalized Hölder's inequality, we have

$$
\begin{aligned}
& \left|f(x)-\frac{\Gamma(1+\alpha)}{(b-a)^{\alpha}}{ }_{a} I_{b}^{\alpha} f(t)\right| \\
\leq & \frac{(x-a)^{2 \alpha}}{(b-a)^{\alpha} \Gamma(1+\alpha)} \int_{0}^{1} t^{\alpha}\left|f^{(\alpha)}(t x+(1-t) a)\right|(d t)^{\alpha} \\
& +\frac{(b-x)^{2 \alpha}}{(b-a)^{\alpha} \Gamma(1+\alpha)} \int_{0}^{1} t^{\alpha}\left|f^{(\alpha)}(t x+(1-t) b)\right|(d t)^{\alpha} \\
\leq & \frac{(x-a)^{2 \alpha}}{(b-a)^{\alpha}}\left(\frac{1}{\Gamma(1+\alpha)} \int_{0}^{1} t^{p \alpha}(d t)^{\alpha}\right)^{\frac{1}{p}}\left(\frac{1}{\Gamma(1+\alpha)} \int_{0}^{1}\left|f^{(\alpha)}(t x+(1-t) a)\right|^{q}(d t)^{\alpha}\right)^{\frac{1}{q}} \\
+ & \frac{(b-x)^{2 \alpha}}{(b-a)^{\alpha}}\left(\frac{1}{\Gamma(1+\alpha)} \int_{0}^{1} t^{p \alpha}(d t)^{\alpha}\right)^{\frac{1}{p}}\left(\frac{1}{\Gamma(1+\alpha)} \int_{0}^{1}\left|f^{(\alpha)}(t x+(1-t) b)\right|^{q}(d t)^{\alpha}\right)^{\frac{1}{q}} .
\end{aligned}
$$

Since $\left|f^{(\alpha)}\right|^{q}$ is generalized $s$-concave on $[a, b]$, applying Theorem 3, we have

$$
\begin{aligned}
\frac{1}{\Gamma(1+\alpha)} \int_{0}^{1}\left|f^{(\alpha)}(t x+(1-t) a)\right|^{q}(d t)^{\alpha} & =\frac{{ }_{a}^{I_{x}^{\alpha}}\left|f^{(\alpha)}(u)\right|}{(x-a)^{\alpha}} \\
& \leq \frac{2^{(s-1) \alpha}}{\Gamma(1+\alpha)}\left|f^{(\alpha)}\left(\frac{a+x}{2}\right)\right|
\end{aligned}
$$

and

$$
\frac{1}{\Gamma(1+\alpha)} \int_{0}^{1}\left|f^{(\alpha)}(t x+(1-t) b)\right|^{q}(d t)^{\alpha} \leq \frac{2^{(s-1) \alpha}}{\Gamma(1+\alpha)}\left|f^{(\alpha)}\left(\frac{b+x}{2}\right)\right| .
$$

If we substitute the inequality (11) and (12), then we obtain the desired result.

Corollary 3. Under assumption of Theorem 7 with $x=\frac{a+b}{2}$, we have the following midpoint inequality

$$
\begin{aligned}
& \left|f\left(\frac{a+b}{2}\right)-\frac{\Gamma(1+\alpha)}{(b-a)^{\alpha}}{ }_{a} I_{b}^{\alpha} f(t)\right| \\
& \leq \frac{(b-a)^{\alpha}}{4^{\alpha}}\left(\frac{2^{(s-1) \alpha}}{\Gamma(1+\alpha)}\right)^{\frac{1}{q}}\left(\frac{\Gamma(1+p \alpha)}{\Gamma(1+(p+1) \alpha)}\right)^{\frac{1}{p}}\left[\left|f^{(\alpha)}\left(\frac{3 a+b}{4}\right)\right|+\left|f^{(\alpha)}\left(\frac{a+3 b}{4}\right)\right|\right]
\end{aligned}
$$

where $p, q>1$ with $\frac{1}{p}+\frac{1}{q}=1$. 


\section{Conclusions}

In this paper, we presented some Ostrowski type inequalities for function whose local fractional derivatives are generalized $s$-convex in the second sense. A further study could be assess similar inequalities by using different types of kernels or convexity.

\section{References}

[1] Budak H., Sarikaya M.Z., Yildirim H., New inequalities for local fractional integrals, Iranian Journal of Science and Technology (Sciences), in press.

[2] Chen G-S., Generalizations of Hölder's and some related integral inequalities on fractal space, Journal of Function Spaces and Applications Volume 2013, Article ID 198405.

[3] Kilıçman A., Saleh W., Notions of generalized s-convex functions on fractal sets, Journal of Inequalities and Applications 2015, 312. DOI 10.1186/s13660-015-0826-x.

[4] Mo H., Sui X., Yu D., Generalized convex functions on fractal sets and two related inequalities, Abstract and Applied Analysis 2014, Article ID 636751, 7 pages.

[5] Mo H., Generalized Hermite-Hadamard inequalities involving local fractional integrals, arXiv:1410.1062 [math.AP].

[6] Mo H., Sui X., Generalized s-convex function on fractal sets, arXiv:1405.0652v2 [math.AP].

[7] Mo H., Sui X., Hermite-Hadamard type inequalities for generalized s-convex functions on real linear fractal set $\mathrm{R}^{\alpha}(0<\alpha<1)$, arXiv:1506.07391v1 [math.CA].

[8] Ostrowski A.M., Über die absolutabweichung einer differentiebaren funktion von ihrem integralmitelwert, Comment. Math. Helv. 1938, 10, 226-227.

[9] Saleh W., Kılıçman A., On generalized s-convex functions on fractal set, JP Journal of Geometry and Topology 2015, 17(1), 63-82.

[10] Sarikaya M.Z., Budak H., Generalized Ostrowski type inequalities for local fractional integrals, RGMIA Research Report Collection 2015, 18, Article 62, 11 p.

[11] Sarikaya M.Z., Erden S., Budak H., Some generalized Ostrowski type inequalities involving local fractional integrals and applications, Advances in Inequalities and Applications 2016, 6.

[12] Sarikaya M.Z., Erden S., Budak H., Some integral inequalities for local fractional integrals, RGMIA Research Report Collection 2015, 18, Article 65, 12 p.

[13] Sarikaya M.Z., Budak H., Erden S., On new inequalities of Simpson's type for generalized convex functions, RGMIA Research Report Collection 2015, 18, Article 66, 13 p.

[14] Yang X.J., Advanced Local Fractional Calculus and Its Applications, World Science Publisher, New York 2012.

[15] Yang J., Baleanu D., Yang X.J., Analysis of fractal wave equations by local fractional Fourier series method, Adv. Math. Phys. 2013, Article ID 632309.

[16] Yang X.J., Local fractional integral equations and their applications, Advances in Computer Science and its Applications (ACSA) 2012, 1(4).

[17] Yang X.J., Generalized local fractional Taylor's formula with local fractional derivative, Journal of Expert Systems 2012, 1(1), 26-30.

[18] Yang X.J., Local fractional Fourier analysis, Advances in Mechanical Engineering and its Applications 2012, 1(1), 12-16.

[19] Yang X.J., Baleanu D., Srivastava H.M., Local Fractional Integral Transforms and their Applications, Elsevier, 2016. 